\title{
Scaling and Universality of ac Conduction in Disordered Solids
}

\author{
Thomas B. Schrøder* and Jeppe C. Dyre \\ Department of Mathematics and Physics (IMFUFA), Roskilde University, Postbox 260, DK-4000 Roskilde, Denmark
}

(Received 2 August 1999)

\begin{abstract}
Recent scaling results for the ac conductivity of ionic glasses by Roling et al. [Phys. Rev. Lett. 78, 2160 (1997)] and Sidebottom [Phys. Rev. Lett. 82, 3653 (1999)] are discussed. We prove that Sidebottom's version of scaling is completely general. A new approximation to the universal ac conductivity arising in the extreme disorder limit of the symmetric hopping model, the "diffusion cluster approximation," is presented and compared to computer simulations and experiments.
\end{abstract}

PACS numbers: 66.30.Dn, 05.60.-k, 72.20.-i

Disordered solids have ac electrical properties remarkably in common [1-6]. These solids, in fact, have so similar frequency-dependent conductivity $\sigma(\omega)$ that ionic conduction cannot be distinguished from electronic. Even the temperature dependence of $\sigma(\omega)$ is "quasiuniversal." The class of disordered solids with quasiuniversal ac behavior is large, including polycrystalline and amorphous semiconductors, organic semiconductors, ionic conductive glasses, ionic viscous melts, nonstoichiometric crystals, ionic or electronic conducting polymers, metal cluster compounds, transition metal oxides, etc. Each class contains hundreds of different solids and there is a huge amount of literature on their ac conductivities.

It is usually possible to scale ac data at different temperatures for one solid into one single curve. This so-called master curve gives the dimensionless ac conductivity $\tilde{\sigma} \equiv$ $\sigma(\omega) / \sigma(0)$ as a function of dimensionless frequency $\tilde{\omega}$. The existence of such a master curve is referred to as the "time-temperature superposition principle" (TTSP).

The common ac features of disordered solids are the following [6]: At low frequencies conductivity is frequency independent. Around the dielectric loss peak frequency $\omega_{m}$ [7] ac conduction sets in, and for $\omega \gg \omega_{m}, \sigma(\omega)$ is close to a frequency power law with exponent $<1.0$. As temperature is lowered, the exponent goes to 1.0. In a log-log plot the ac conductivity is much less temperature dependent than the dc conductivity. A final ubiquitous observation is the Barton-Nakajima-Namikawa (BNN) relation [2,8-11] connecting dielectric loss peak frequency, dielectric loss strength $\Delta \epsilon$ [7], and dc conductivity: $\sigma(0)=p \Delta \epsilon \epsilon_{0} \omega_{m}$, where $p$ is a numerical constant of order 1 .

To construct the ac master curve frequency must be divided by $\omega_{m}$. Because the dielectric loss strength is only weakly temperature dependent while $\sigma(0)$ and $\omega_{m}$ are both Arrhenius, the BNN relation implies $\omega_{m} \sim \sigma(0)$. Thus, the existence of a master curve is conveniently summarized into

$$
\tilde{\sigma}=F\left(\frac{C}{\sigma(0)} \omega\right)
$$

where $C$ may depend on variables like charge carrier concentration $n$, temperature $T$, high frequency dielectric constant, etc.
In a series of papers towards the end of the 1950s Taylor analyzed the dielectric properties of ionic glasses in accordance with the Debye equation with a spread of relaxation times [12]. He showed that the dielectric loss [7] for all glasses fell on a single plot against scaled frequency. In 1961 Isard relabeled Taylor's axis by plotting dielectric loss against log of the product of frequency and resistivity and thus essentially arrived at ac scaling in the form given in Eq. (1) [13]. Since then Eq. (1), which we shall term "Taylor-Isard scaling," has been used for several ionic as well as electronic conducting disordered solids to construct ac master curves from measurements at different temperatures [14-22].

Recently, there has been renewed interest in scaling and universality of ac data for ionic conductive glasses [22-26]. In 1997 Roling, Happe, Funke, and Ingram showed that the Taylor-Isard scaling constant $C$ is proportional to $n / T$ for sodium borate glasses [23]. Last year, however, Sidebottom showed that in general scaling is not achieved by $C \propto n / T$; instead two different ionic conductive glasses and one highly viscous ionic liquid just above the glass transition all obey the following scaling relation [25]:

$$
\tilde{\sigma}=F\left(\frac{\epsilon_{0} \Delta \epsilon}{\sigma(0)} \omega\right)
$$

In this Letter we prove that Eq. (2) is correct whenever scaling is possible at all (TTSP obeyed). A new analytical approximation to the universal ac conductivity of the so-called symmetric hopping model is presented and compared to computer simulations. Finally, the experimental master curves discussed by Sidebottom are compared to the universal ac hopping conductivity.

First, however, we comment on the scaling principle of Roling and co-workers, $C \propto n / T$ in Eq. (1) [23], and Sidebottom's interpretation of its occasional violations [25]. If $q$ is charge, $f_{H}$ jump rate, and $d$ jump length, Sidebottom bases his arguments on the expressions $\sigma(0) \propto$ $n q^{2} d^{2} f_{H} / k_{B} T$ and $\Delta \epsilon \propto n q^{2} d^{2} / k_{B} T$. Clearly, to obtain $\tilde{\omega}$ frequency should be divided by $f_{H}$, leading to $C \propto n / T$ if the jump length $d$ is constant. Since $d$ could change as concentration changes, Sidebottom argues that in general one cannot expect $C \propto n / T$ but should rather have 
$C \propto n d^{2} / T$ - the potential $n$ dependence of $d$ is, however, taken care of by Eq. (2) in which the unknown jump length is eliminated by scaling with the measured $\Delta \epsilon$.

In our opinion Sidebottom's arguments are largely correct, but Eq. (2) is much more general than it appears from his reasoning. Consider hopping of completely noninteracting charge carriers on a cubic lattice. In this model conductivity is $n q$ times charge carrier mobility and for given lattice jump frequencies the mobility scales with $d^{2}$, as Sidebottom has it. However, if the lattice is homogeneous, with just one jump frequency, conductivity is frequency independent - in order to have strongly frequency-dependent conductivity, lattice jump frequencies must cover many decades. In this case the expressions used by Sidebottom for $\sigma(0)$ and $\Delta \epsilon$ do not apply - there simply is no unique jump rate $f_{H}$. Still, we find in our simulations that Eq. (2) is obeyed. This leads to the question: When does Eq. (2) apply?

Equation (2) apparently expresses two pieces of information: (a) TTSP is obeyed, and (b) the scaled frequency is given by $\tilde{\omega}=\left[\epsilon_{0} \Delta \epsilon / \sigma(0)\right] \omega$. We now prove, however, that (a) mathematically implies (b): TTSP implies the existence of some function $\tilde{\sigma}(\tilde{\omega})$ where $\tilde{\omega}$ is the scaled frequency. Expanding to first order in $\tilde{\omega}$ leads to $\tilde{\sigma}=$ $1+i \tilde{\omega} A$ [where $A$ is real because $\sigma^{*}(\omega)=\sigma(-\omega)$ ]. Since $\sigma=\tilde{\sigma} \sigma(0)$ we have $\sigma=\sigma(0)+i \tilde{\omega} A \sigma(0)$ for $\omega \rightarrow 0$. On the other hand, from the definition of $\Delta \epsilon$ [7] one has $\sigma=\sigma(0)+i \omega \Delta \epsilon \epsilon_{0}$ for $\omega \rightarrow 0$. Equating these two asymptotic expressions for $\sigma$ leads to $\tilde{\omega}=$ $A^{-1}\left[\Delta \epsilon \epsilon_{0} / \sigma(0)\right] \omega$. We have thus shown that $\tilde{\sigma}$ is a function of $A^{-1}\left[\Delta \epsilon \epsilon_{0} / \sigma(0)\right] \omega$. This implies that $\tilde{\sigma}$ is a function of $\left[\Delta \epsilon \epsilon_{0} / \sigma(0)\right] \omega$, i.e., Eq. (2) [27]. In practice the scaling expressed by Eq. (2) simply means that frequency is scaled such that the imaginary part of the normalized ac conductivity is equal to scaled frequency as $\omega \rightarrow 0$.

A simple model for ac conduction in disordered solids is the symmetric hopping model [28-32]. It considers random walks of noninteracting particles on a cubic lattice with random symmetric Arrhenius nearest-neighbor jump rates $\left(\gamma_{0} e^{-\beta E}\right.$, where $\beta=1 / k_{B} T$ and $E$ is the spatially randomly varying energy barrier). According to the effective medium approximation (EMA) this model approaches universality in the extreme disorder limit: As $\beta \rightarrow \infty$, $\tilde{\sigma}$ as a function of a well-defined scaled frequency $\tilde{\omega}$ becomes independent of both $\beta$ and of the energy barrier probability distribution [31]. This predicted ac universality was confirmed by computer simulations in two dimensions at imaginary frequencies, although the EMA universality prediction $\tilde{\sigma} \ln \tilde{\sigma}=i \tilde{\omega}$ turned out to be inaccurate [31]. In Fig. 1 we present results from computer simulations of the symmetric hopping model in three dimensions at real frequencies. These simulations were done on samples with periodic boundary conditions solving the master equation utilizing a new algorithm [33]. Figure 1a shows $\tilde{\sigma}^{\prime}$-the real part of $\tilde{\sigma}$ - for the box distribution of energy barriers. The frequency axis is scaled according to Eq. (2). As $\beta$ increases, $\tilde{\sigma}^{\prime}$ converges to a single curve. Figure $1 \mathrm{~b}$ shows
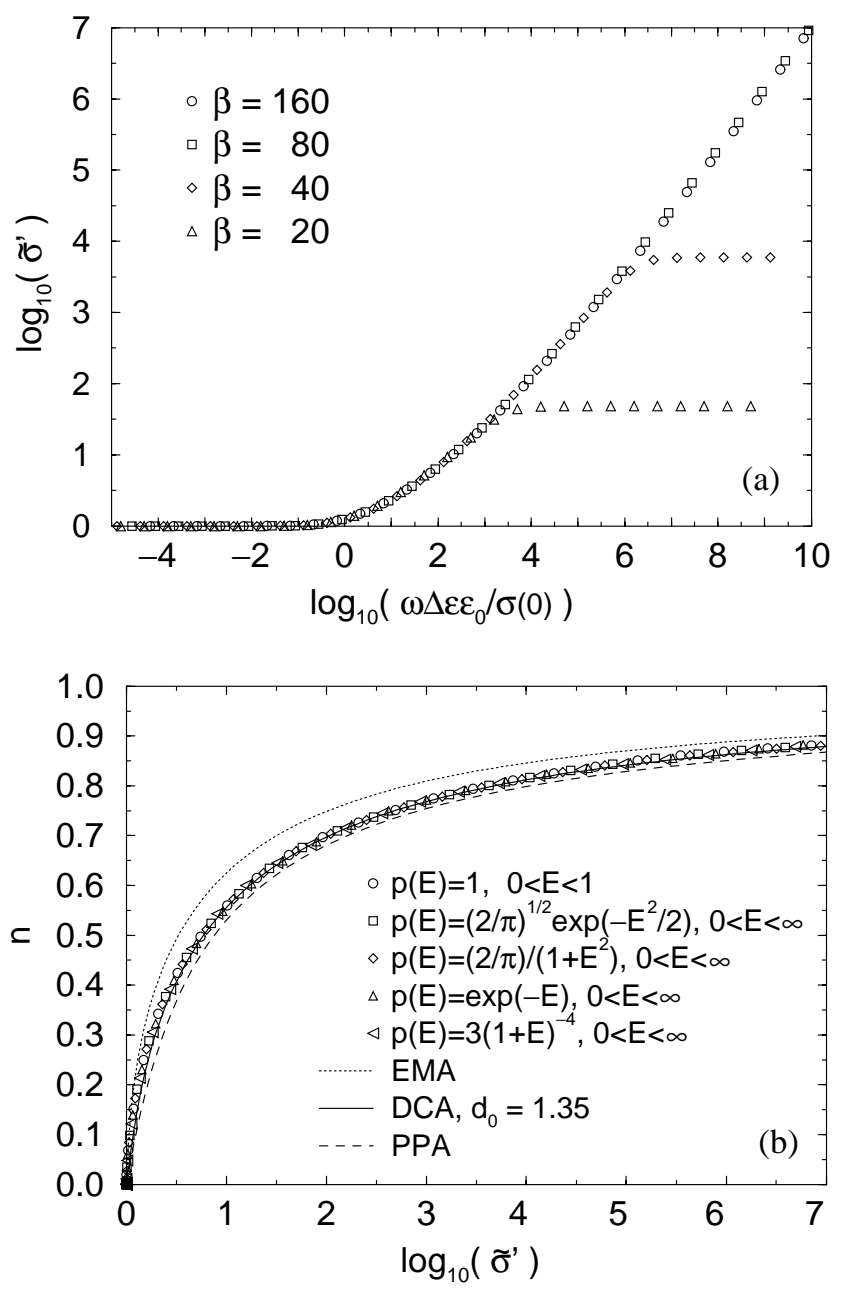

FIG. 1. Numerical results for the symmetric hopping model in three dimensions with periodic boundary conditions [33]. Reported results are averages over 100 different $N \times N \times N$ cubic lattices. (a) Real part of the dimensionless ac conductivity, $\tilde{\sigma}^{\prime}(\tilde{\omega})$, for the box distribution of energy barriers $[p(E)=$ $1,0 \leq E \leq 1$ ], where $\tilde{\omega}$ is given by Eq. (2); $\beta=20(N=14)$, $\beta=40(N=24), \beta=80(N=32)$, and $\beta=160(N=64)$. As $\beta$ increases, the data converge to a universal curve. (b) The apparent exponent $n \equiv d \ln \tilde{\sigma}^{\prime} / d \ln \tilde{\omega}$ plotted versus $\tilde{\sigma}^{\prime}$. Data are shown for five different energy barrier distributions at $\beta$ 's given by $\beta=160 p\left(E_{c}\right)(N=64)$ where $E_{c}$ is the "percolation energy" [34,37]. The universal curve is independent of the energy barrier distribution. The numerical data are compared to three analytical approximations: EMA $(\tilde{\sigma} \ln \tilde{\sigma}=i \tilde{\omega}$ [31]), PPA $[\ln (1+\sqrt{i \tilde{\omega} \tilde{\sigma}})=\sqrt{i \tilde{\omega} / \tilde{\sigma}}$ [34]], and DCA with $d_{0}=1.35$ [Eq. (3)]. The universal curve lies between EMA and PPA and is well approximated by DCA with $d_{0}=1.35$.

the apparent exponent $n \equiv d \ln \tilde{\sigma} / d \ln \tilde{\omega}$ as a function of $\tilde{\sigma}^{\prime}$ for different energy barrier probability distributions at large $\beta$ 's, proving ac universality for the hopping model. Figure $1 \mathrm{~b}$ also compares the simulations to three analytical approximations, the EMA universality equation $\tilde{\sigma} \ln \tilde{\sigma}=i \tilde{\omega}[29,31]$, the percolation path approximation (PPA) [34-36], and a new phenomenological "diffusion cluster approximation" (DCA), which we describe below.

In the extreme disorder limit hopping conduction takes place on the "percolation cluster" formed from lattice 
links with largest jump rates until percolation [31,37]. The PPA idea $[34,37]$ is to regard the conducting paths on the percolation cluster as strictly one dimensional. On the other hand, the idea behind EMA is to replace the inhomogeneous lattice by an effective homogeneous medium determined self-consistently [29,30]. Our hopping simulations show that $\tilde{\sigma}^{\prime}(\tilde{\omega})$ is somewhere between PPA and EMA. Most likely, this is because both approximations ignore the fact that conduction takes place on some complex subset of the percolation cluster. This "diffusion cluster" must be smaller than the backbone (defined by removing dead ends of the percolation cluster, fractal dimension $=1.7[33,38])$ and larger than the set of red bonds (those that, when cut, stop the current, fractal dimension $=1.1[33,38])$. At present this is all we know about the diffusion cluster, and its dimension $d_{0}$ is regarded below as a fitting parameter. To derive the DCA equation we use EMA in $d_{0}$ dimensions. In the extreme disorder limit EMA implies $[31,39] \ln \tilde{\sigma} \propto s \tilde{G}$ where $s \tilde{G}$ is defined by $(2 \pi)^{d_{0}} s \tilde{G}=$ $\int_{-\pi<k_{i}<\pi} d \mathbf{k}\left\{i \omega /\left(i \omega+2 \sigma\left[d_{0}-\sum \cos k_{i}\right]\right)\right\}$. Whenever $1<d_{0}<2$ one finds $s \tilde{G} \propto(i \omega / \sigma)^{d_{0} / 2}$ at relevant [31] frequencies. Thus, after rescaling frequency we arrive at the DCA equation,

$$
\ln \tilde{\sigma}=\left(\frac{i \tilde{\omega}}{\tilde{\sigma}}\right)^{d_{0} / 2} .
$$

As is clear from Fig. 1b, the solution to this equation for $d_{0}=1.35$ gives an excellent fit to the universal ac hopping conductivity.

In Fig. 2 the three sets of data discussed by Sidebottom [25] are compared to the hopping model simulations in the extreme disorder limit (filled squares) and to the DCA (full line). For all three systems we find a frequency range where experimental data agree well with the symmetric hopping model.
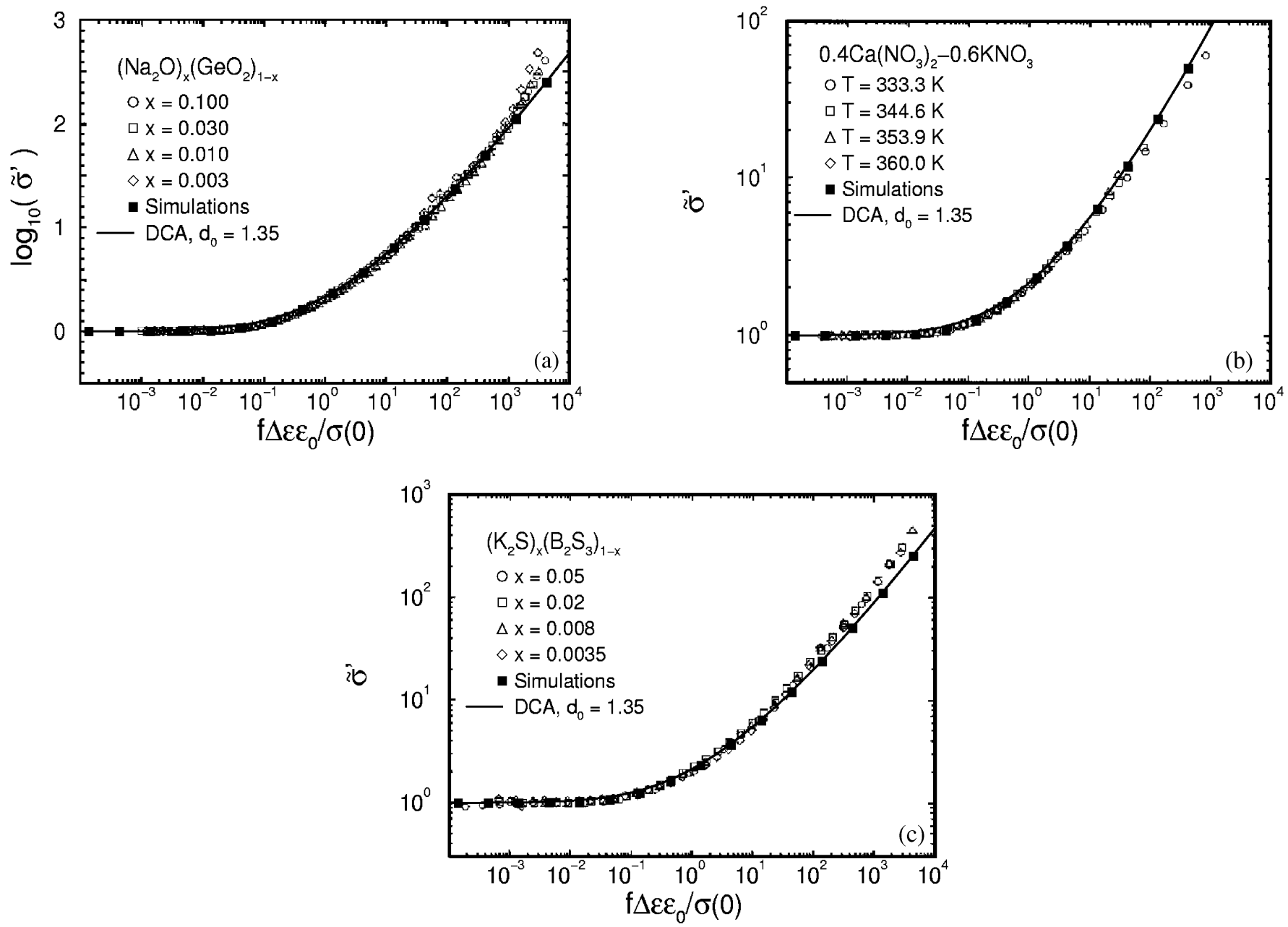

FIG. 2. Experimental data for ac conduction of three ionic systems scaled according to Eq. (2) $(f=\omega / 2 \pi)$ (reproduced from Ref. [25]): (a) $\left(\mathrm{Na}_{2} \mathrm{O}\right)_{x}\left(\mathrm{GeO}_{2}\right)_{1-x}$ (Sidebottom [25]); (b) 0.4Ca( $\left.\mathrm{NO}_{3}\right)_{2}-0.6 \mathrm{KNO}_{3}$ (Howell et al. [40]); (c) $\left(\mathrm{K}_{2} \mathrm{~S}\right)_{x}\left(\mathrm{~B}_{2} \mathrm{~S}_{3}\right)_{1-x}(\mathrm{Patel}$ [41]). The experimental data are compared to the universal ac conductivity of the symmetric hopping model (numerical data, full squares) and DCA [Eq. (3) with $d_{0}=1.35$ ]. [The DCA prediction is empirically scaled to agree with the numerical data for the hopping model; it cannot be scaled using Eq. (2) since $\Delta \epsilon_{\mathrm{DCA}}=\infty$, a low-frequency artifact of this approximation.] Comparing computer simulations to the experimental data it should be noted that there are no adjustable parameters: In the extreme disorder limit the model predictions become independent of both $\beta$ and of energy barrier probability distribution. 
The symmetric hopping model is a highly idealized model. The model ignores Coulomb repulsions and it allows an arbitrary number of charge carriers at each site. This is unrealistic, but it should be noted that the model is mathematically identical to that obtained by linearizing (with respect to the electric field) a hopping model with energy disorder and self-exclusion (i.e., Fermi statistics) [29].

To summarize, we have proved that Sidebottom's scaling version Eq. (2) applies whenever scaling is possible at all (TTSP obeyed), i.e., that the Taylor-Isard scaling constant $C$ of Eq. (1) is always proportional to $\Delta \epsilon$. A new analytical approximation to the universal ac conductivity of hopping in the extreme disorder limit, the diffusion cluster approximation (DCA), has been presented and shown to give an excellent fit to simulations. Finally, we have shown that DCA (and thereby the extreme disorder limit of the symmetric hopping model) agrees well with the three sets of ionic data discussed by Sidebottom [25] in a frequency range depending on the system.

*Present address: Center for Theoretical and Computational Materials Science, National Institute of Standards and Technology, Gaithersburg, MD 20899.

[1] J. O. Isard, J. Non-Cryst. Solids 4, 357 (1970).

[2] H. Namikawa, J. Non-Cryst. Solids 18, 173 (1975).

[3] A. K. Jonscher, Nature (London) 267, 673 (1977); Universal Relaxation Law (Chelsea Dielectric, London, 1996).

[4] A. E. Owen, J. Non-Cryst. Solids 25, 372 (1977).

[5] A. Mansingh, Bull. Mater. Sci. (India) 2, 372 (1980).

[6] J. C. Dyre, J. Appl. Phys. 64, 2456 (1988).

[7] If the free charge carrier ac conductivity is $\sigma(\omega)$, the (relative) dielectric constant $\epsilon(\omega)$ is defined by $\sigma(\omega)-$ $\sigma(0)=i \omega\left[\epsilon(\omega)-\epsilon_{\infty}\right] \epsilon_{0}$ where $\epsilon_{\infty}$ is the high frequency (relative) dielectric constant and $\epsilon_{0}$ is the vacuum permittivity. Writing $\epsilon(\omega)=\epsilon^{\prime}(\omega)-i \epsilon^{\prime \prime}(\omega)$, the quantity $\epsilon^{\prime \prime}(\omega)>0$ is referred to as the dielectric loss. The loss peak frequency $\omega_{m}$ is the frequency where $\epsilon^{\prime \prime}(\omega)$ reaches its maximum. The dielectric loss strength $\Delta \epsilon$ is $\epsilon(0)-\epsilon_{\infty}$.

[8] J. L. Barton, Verres Réfract. 20, 328 (1966).

[9] T. Nakajima, in 1971 Annual Report, Conference on Electric Insulation and Dielectric Phenomena (National Academy of Sciences, Washington, DC, 1972), p. 168.

[10] M. Tomozawa, in Treatise on Materials Science, edited by M. Tomozawa (Academic, New York, 1977), Vol. 12, p. 283.

[11] J. C. Dyre, J. Non-Cryst. Solids 88, 271 (1986).

[12] H. E. Taylor, Trans. Faraday Soc. 41, 350 (1956); J. Soc. Glass Technol. 41, 350T (1957); 43, 124T (1959).

[13] J. O. Isard, Proc. Inst. Electr. Eng. 109B, Suppl. No. 22, 440 (1961) (Paper No. 3636).

[14] H. Scher and M. Lax, Phys. Rev. B 7, 4491 (1973); Phys. Rev. B 7, 4502 (1973).

[15] S. Summerfield, Philos. Mag. B 52, 9 (1985).

[16] N. Balkan, P. N. Butcher, W. R. Hogg, A. R. Long, and S. Summerfield, Philos. Mag. B 51, L7 (1985).
[17] W. Rehwald, H. Kiess, and B. Binggeli, Z. Phys. B 68, 143 (1987).

[18] M. P. J. van Staveren, H. B. Brom, and L. J. de Jongh, Phys. Rep. 208, 1 (1991).

[19] H. Kahnt, Ber. Bunsen-Ges. Phys. Chem. 95, 1021 (1991).

[20] B. Fiegl, R. Kuhnert, M. Ben-Chorin, and F. Koch, Appl. Phys. Lett. 65, 371 (1994).

[21] A. R. Kulkarni, P. Lunkenheimer, and A. Loidl, Solid State Ion. 112, 69 (1998).

[22] B. Roling, Solid State Ion. 105, 185 (1998).

[23] B. Roling, A. Happe, K. Funke, and M. D. Ingram, Phys. Rev. Lett. 78, 2160 (1997).

[24] K. Funke and C. Cramer, Curr. Opin. Solid State Mater. Sci. 2, 483 (1997).

[25] D. L. Sidebottom, Phys. Rev. Lett. 82, 3653 (1999).

[26] A. Ghosh and M. Sural, Europhys. Lett. 47, 688 (1999).

[27] Besides TTSP the only assumption made is analyticity or, more precisely, the existence of a regular first order term in the expansion of $\tilde{\sigma}(\tilde{\omega})$. Note that, while the existence of this regular first order term is rarely disputed, the low-frequency expansion of the ac conductivity looks like this in hopping models: $\sigma(\omega)=\sigma(0)+a(i \omega)+$ $b(i \omega)^{3 / 2}+\ldots$ (the nonanalyticity is a consequence of the existence of long time tails for random walks in random structures [29]). Note also that the BNN relation follows from Eq. (2): Since $\epsilon(\omega)-\epsilon_{\infty} \propto(\tilde{\sigma}-1) / \tilde{\omega}$ is a function of $\tilde{\omega}$ the dielectric loss has its maximum at some particular temperature-independent value of $\tilde{\omega}$. Denoting this value by $\tilde{\omega}_{m}$ we have $\tilde{\omega}_{m}=A^{-1}\left[\Delta \epsilon \epsilon_{0} / \sigma(0)\right] \omega_{m}$. If one defines $p=1 / A \tilde{\omega}_{m}$ we find $1 / p=\left[\Delta \epsilon \epsilon_{0} / \sigma(0)\right] \omega_{m}$ which is the $\mathrm{BNN}$ relation.

[28] J. C. Kimball and L.W. Adams, Phys. Rev. B 18, 5851 (1978).

[29] H. Böttger and V.V. Bryksin, Hopping Conduction in Solids (Akademie-Verlag, Berlin, 1985).

[30] J. W. Haus and K. W. Kehr, Phys. Rep. 150, 263 (1987).

[31] J. C. Dyre, Phys. Rev. B 49, 11709 (1994).

[32] D. L. Stein and C. M. Newman, Phys. Rev. E 51, 5228 (1995).

[33] T. B. Schrøder, Ph.D. thesis, Roskilde University, 1999.

[34] J. C. Dyre and T.B. Schrøder, Phys. Rev. B 54, 14884 (1996).

[35] The percolation path approximation [34] gives the following equation for the universal ac hopping conductivity: $\ln (1+\sqrt{i \tilde{\omega} \tilde{\sigma}})=\sqrt{i \tilde{\omega} / \tilde{\sigma}}$.

[36] In Ref. [34] the universal curve was found to be slightly closer to PPA than in Fig. 1b. This minor difference is due to different boundary conditions used. We believe that compared to Ref. [34] the present periodic boundary conditions give results which for any finite lattice are closer to the true bulk conductivity.

[37] J. C. Dyre, Phys. Rev. B 48, 12511 (1993).

[38] D. Stauffer and A. Aharoni, Introduction to Percolation Theory (Taylor and Francis, London, 1992), 2nd ed.

[39] Rationalized units are used here (i.e., conductivity = jump rate on a homogeneous lattice) [31].

[40] F. S. Howell, R. A. Bose, P. B. Macedo, and C. T. Moynihan, J. Phys. Chem. 78, 639 (1974).

[41] H. K. Patel, Ph.D. thesis, Iowa State University, Ames, IA, 1993. 